\title{
A nonadiabatic oscillation study of DB white dwarfs
}

\author{
A. Gautschy ${ }^{1}$ and L. G. Althaus ${ }^{2}$ \\ 1 Ferrachstr. 1, 8630 Rüti, Switzerland \\ 2 Facultad de Ciencias Astronómicas y Geofisicas, Universidad Nacional de la Plata, Paseo del Bosque S/N, \\ 1900 La Plata, Argentina, Member of the Carrera del Investigador Científico y Tecnológico (CONICET)
}

Received 17 July 2001 / Accepted 7 November 2001

\begin{abstract}
A self-consistent abundance diffusion treatment in the evolution of cooling white dwarfs permitted a study of the effect of elemental segregation in nonadiabatic, nonradial stability computations. In particular, mode trapping manifesting itself in cyclically varying period separations behaved differently from its appearance in the damping/excitation rates. Another aspect of the investigation concerned the effect of heavy-element traces in homogeneous DB white-dwarf envelopes on their pulsational instability domain. The stellar models are computed with the CGM convection approach; the study can therefore be considered as a test of its performance in nonradial stability analyses.
\end{abstract}

Key words. stars: oscillations - stars: white dwarfs - stars: interior

\section{Introduction}

The variable white dwarfs of the various spectroscopic flavors (i.e. DOV, DBV, and DAV; e.g. Gautschy \& Saio 1996) belong currently to the best studied oscillating stars. Long-term observing strategies, such as WET (Nather 1995), the seismological promises, and eventually their successes (e.g. Kawaler 1998, and references therein) are the pillars of the scientific progress in this field.

To seismologically interpret the rich observed oscillation-mode spectra, adiabatic pulsation theory proved successful (e.g. Bradley et al. 1993; Bradley 1996). The computed adiabatic eigenmodes and in particular the period separations between neighboring overtones and the deviations from equi-spacing were developed into the main diagnostic tool to infer masses and magnitudes of the material layers in the compositionally stratified pulsators. Frequency splittings even opened the door to attempts to study the spatial structure of white dwarfs' rotation profiles (Kawaler et al. 1999).

Adiabatic theory, however, cannot determine which and the number of pulsation modes that are eventually excited and therefore at least potentially observable. This second step requires at least nonadiabatic modeling. For various reasons, the level of reliability of nonadiabatic results is not as high as for the adiabatic ones. A

Send offprint requests to: A. Gautschy, e-mail: althaus@f caglp.unlp.edu .ar major obstacle in the numerical realizations is the small growth/damping rates found for white dwarfs' $g$ modes and the close proximity to adiabaticity of the thermodynamic processes already close to the surface.

Considering the position of the DB white dwarfs on the Hertzsprung-Russell (HR) plane, we recognize that they populate the same effective-temperature range - though at much lower luminosity - as the $\beta$ Cephei variables. For the instability of the latter, the $Z$-bump in the OPAL/OP opacities (e.g. Iglesias \& Rogers 1996; Seaton et al. 1994) is accepted to provide the necessary driving through the classical $\kappa$ mechanism. Despite the $Z$-bump getting less pronounced at the higher densities which are relevant for white dwarfs, it is a priori unclear if remaining traces of heavy elements in the envelopes of cooling white dwarfs influence the position of the blue edge of the pulsation domain of the DBV class. The initial question for this study was therefore: how do heavy elements in the envelopes of DB white dwarfs influence their pulsational stability?

As a detailed diffusion treatment of nuclear species in the evolutionary star models became available during this project, we extended its scope and computed various model series to study the trapping properties of nonadiabatic pulsation modes in DBVs with diffusively evolving stratifications. The studied model sequences are listed in Table 1 . The nonadiabatic stability results are presented in Sect. 3. Section 4 contains a detailed discussion, mainly of the trapping results. We conclude the paper with a 
speculation on the longstanding problem of encountering too many overstable linear modes as compared with the observed oscillation spectra.

\section{Methods and models}

\subsection{Stellar structure and evolution}

The stellar-evolution code used in this work is the same as the one employed in the recent studies of WD evolution by Althaus \& Benvenuto $(1997,2000)$ and Benvenuto \& Althaus (1998); it is based on the method of Kippenhahn et al. (1967). In particular, to specify the outer boundary conditions we carry out three envelope integrations (at constant luminosity) from photospheric starting values inward to a fitting outer mass fraction of $m_{1} / M_{*} \approx 10^{-16}$ where $m_{1}$ corresponds to the first mass shell in the Henyey scheme and $M_{*}$ is the total mass of the model. The interior solution is obtained via the canonical Henyey iteration scheme as described by Kippenhahn et al. (1967). Our stellar models were divided into $\approx 2000$ mesh points, most of them distributed in the outer layers. Concerning constitutive physics, the code is based on a very detailed and updated physical description such as OPAL radiative opacities (Iglesias \& Rogers 1996) for different metalicities consistent with expectations from element diffusion. We also consider the full-spectrum turbulence theory of convection developed by Canuto et al. (1996) (referred to as CGM in the following). Figure 1 shows the extent of the outer convection zone in mass, parameterized by $q \equiv 1-m / M_{*}$, where $m$ stands for the mass, as a function of effective temperature for the diffusion-free, $Z=0$ DB models with $0.6 M_{\odot}$ (sequence I6 in Table 1). The grey area depicts the spatial evolution along the white dwarf's cooling track of the surface convection computed with the CGM model. The heavy lines indicate the convective boundaries as computed with an ML2 formulation (cf. Tassoul et al. 1990) assuming a mixing length of one pressure scale height.

The equation of state (EoS) for the low-density regime is an updated version of that of Magni \& Mazzitelli (1979), while for the high density regime we consider ionic contributions, Coulomb interactions, partially degenerate electrons, electron exchange, and Thomas-Fermi contributions at finite temperature. High-density conductive opacities and the various rates of neutrino emission (photo-, plasma-, and Bremsstrahlung neutrinos) are adopted from the works of Itoh and collaborators (see Althaus \& Benvenuto 1997 for details).

One important aspect of the present study is the explicit accounting of the evolution of the chemical abundance distribution due to diffusion processes for some of the model sequences. We considered gravitational settling (pressure diffusion), chemical, and thermal diffusion of nuclear species. To this end, we adopted the treatment for multicomponent gases presented by Burgers (1969), thus avoiding the trace element approximation usually invoked in most WD studies. Radiative levitation, which is

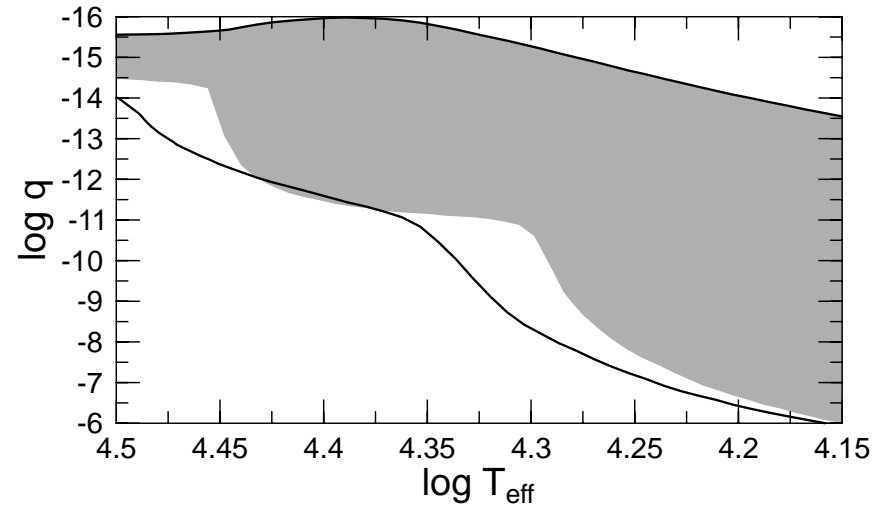

Fig. 1. Extent in mass of the outer convection zone in $Z=$ $0,0.6 M_{\odot}$ DB white dwarf models. The grey area shows the magnitude of the convection zone as computed with the CGM formalism. The outer and inner convective boundaries, as obtained with an ML2 computation (with a mixing length of one pressure scale height) are drawn as heavy lines.

important to determine photospheric composition of hot WDs was neglected. This assumption is justified since we are interested in the chemical evolution occurring quite deep in the star. In the context of WD evolution, the treatment of diffusion we use here has been employed by Iben \& MacDonald (1985). Recently, it was applied by MacDonald et al. (1999) to address the problem of carbon dredge-up in WDs with helium-rich envelopes and by Althaus et al. (2001) to analyze the role played by diffusion to induce thermonuclear flashes in low-mass, heliumcore WDs. To the best of our knowledge, only Dehner \& Kawaler (1995) used also non-equilibrium diffusion star models for - in their case adiabatic - pulsation studies.

As a result of gravity, partial pressure, thermal gradients and induced electric fields (we neglect stellar rotation and magnetic fields) the diffusion velocities in a multicomponent plasma satisfy the set of diffusion equations $(N-1$ independent linear equations, Burgers 1969)

$$
\begin{aligned}
\frac{\mathrm{d} p_{i}}{\mathrm{~d} r} & -\frac{\rho_{i}}{\rho} \frac{\mathrm{d} p}{\mathrm{~d} r}-n_{i} Z_{i} e E=\sum_{j \neq i}^{N} K_{i j}\left(w_{j}-w_{i}\right) \\
& +\sum_{j \neq i}^{N} K_{i j} z_{i j} \frac{m_{j} r_{i}-m_{i} r_{j}}{m_{i}+m_{j}}
\end{aligned}
$$

and heat flow equations ( $N$ equations)

$$
\begin{aligned}
& \frac{5}{2} n_{i} k_{\mathrm{B}} \nabla T=-\frac{5}{2} \sum_{j \neq i}^{N} K_{i j} z_{i j} \frac{m_{j}}{m_{i}+m_{j}}\left(w_{j}-w_{i}\right) \\
& -\frac{2}{5} K_{i i} z_{i i}^{\prime \prime} r_{i} \\
& -\sum_{j \neq i}^{N} \frac{K_{i j}}{\left(m_{i}+m_{j}\right)^{2}}\left(3 m_{i}^{2}+m_{j}^{2} z_{i j}^{\prime}+0.8 m_{i} m_{j} z_{i j}^{\prime \prime}\right) r_{i} \\
& +\sum_{j \neq i}^{N} \frac{K_{i j} m_{i} m_{j}}{\left(m_{i}+m_{j}\right)^{2}}\left(3+z_{i j}^{\prime}-0.8 z_{i j}^{\prime \prime}\right) r_{j}
\end{aligned}
$$


In these equations, $p_{i}, \rho_{i}, n_{i}, Z_{i}$ and $m_{i}$ means, respectively, the partial pressure, mass density, number density, mean charge and mass for species $i$ ( $N$ means the number of ionic species plus electron). The quantities $T$ and $k_{\mathrm{B}}$ are the temperature and the Boltzmann constant. The unknown variables are the diffusion velocities with respect to the center of mass, $w_{i}$, and the residual heat flows $r_{i}$ (for ions and electrons). The electric field $E$ has also to be determined. The resistance coefficients $\left(K_{i j}, z_{i j}, z_{i j}^{\prime}\right.$ and $\left.z_{i j}^{\prime \prime}\right)$ are adapted from Paquette et al. (1986a). Average ionic charges are treated following an approximate pressureionization model as given by Paquette et al. (1986b) which is sufficient for our purposes.

To complete the set of equations, we use the conditions for a vanishing net mass flow with respect to the center of mass

$\sum_{i} A_{i} n_{i} w_{i}=0$

and vanishing electrical current

$\sum_{i} Z_{i} n_{i} w_{i}=0$

In terms of the gradient in the number density we can transform Eq. (1) to

$$
\begin{aligned}
\frac{1}{n_{i}}\left[\sum_{j \neq i}^{N} K_{i j}\left(w_{i}-w_{j}\right)\right. & \left.+\sum_{j \neq i}^{N} K_{i j} z_{i j} \frac{m_{i} r_{j}-m_{j} r_{i}}{m_{i}+m_{j}}\right] \\
& -Z_{i} e E=\alpha_{i}-k_{\mathrm{B}} T \frac{\mathrm{d} \ln n_{i}}{\mathrm{~d} r},
\end{aligned}
$$

where

$\alpha_{i}=-A_{i} m_{\mathrm{H}} g-k_{\mathrm{B}} T \frac{\mathrm{d} \ln T}{\mathrm{~d} r}$,

with $A_{i}, m_{\mathrm{H}}$, and $g$ being the atomic mass number, the hydrogen-atom mass, and gravity, respectively. We write the unknowns $w_{i}, r_{i}$ and $E$ in terms of the gradient of ion densities in the form (similarly for $r_{i}$ and $E$ )

$w_{i}=w_{i}^{\mathrm{gt}}-\sum_{\operatorname{ions}(j)} \sigma_{i j} \frac{\mathrm{d} \ln n_{j}}{\mathrm{~d} r}$,

where $w_{i}^{\text {gt }}$ stands for the velocity component due to gravitational settling and thermal diffusion. The summation in Eq. (7) is to be effected over the ions only. With Eqs. (2) and (5), together with (3) and (4), we can find the components $w_{i}^{\mathrm{gt}}$ and $\sigma_{i j}$ by matrix inversions.

To find the evolution of the abundance distribution throughout the star we solve the elemental continuity equations. Details are given in Althaus \& Benvenuto (2000). In particular, we follow the evolution of the isotopes ${ }^{4} \mathrm{He},{ }^{12} \mathrm{C}$, and ${ }^{16} \mathrm{O}$. In order to calculate the dependence of the structure of our WD models on the varying abundances self-consistently, the set of equations describing diffusion has been coupled to the evolutionary code. After computing the change of abundances by the effect of diffusion, they are evolved according to the requirements

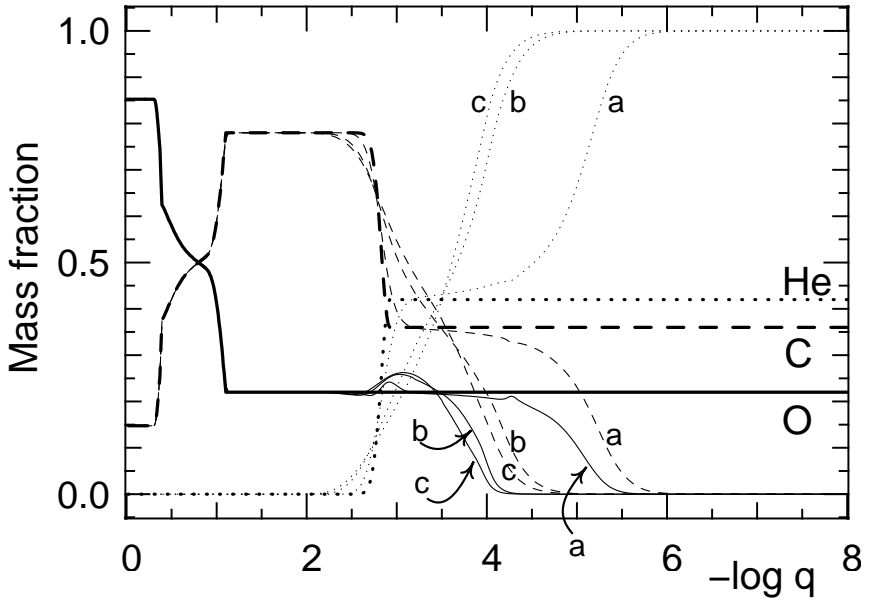

Fig. 2. Exemplary diffusive abundance evolution in a $0.6 M_{\odot}$ model with an initial He envelope of $6.6 \times 10^{-4} M_{*}$. Initial profiles for $\mathrm{He}, \mathrm{C}$, and $\mathrm{O}$ are plotted with heavy lines. Later stages are each labeled with a $\left(\log T_{\text {eff }}=4.454\right), \mathrm{b}\left(\log T_{\text {eff }}=\right.$ $4.275)$, and $\mathrm{c}\left(\log T_{\mathrm{eff}}=4.206\right)$.

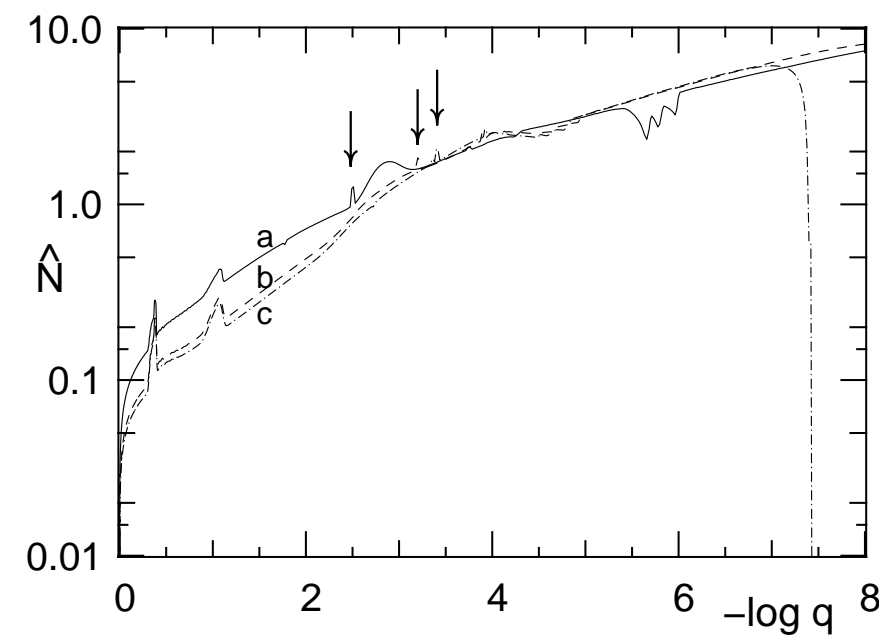

Fig. 3. Free-fall normalized Brunt-Väisälä frequencies, $\hat{N}$, for models a, b, and c, whose composition profiles are displayed in Fig. 2. The vertical arrows indicate the numerically inflicted $\hat{N}$-spike discussed in the text.

of convective mixing. Finally, we emphasize that radiative opacities are calculated for metalicities consistent with the diffusion predictions.

An example of a typical profile evolution is shown in Fig. 2. The selected white-dwarf model has $0.6 M_{\odot}$ and a helium envelope of $6.6 \times 10^{-4} M_{*}$. The initial profiles for $\mathrm{He}, \mathrm{C}$, and $\mathrm{O}$ are plotted with heavy lines having various patterns. The profiles at evolutionary stages around the DBV instability domain are labeled with the letters a (at $\left.\log T_{\text {eff }}=4.454\right)$, b (at $\left.\log T_{\text {eff }}=4.275\right)$, and $\mathrm{c}\left(\right.$ at $\left.\log T_{\text {eff }}=4.206\right)$. Figure 3 shows the corresponding, free-fall normalized Brunt-Väisälä frequencies for the three models. The diffusion-induced helium depletion and carbon and oxygen enhancement in the envelope leave easily recognizable traces in the Brunt-Väisälä frequency only in model a for which this region is still 
Table 1. Model sequences used for nonadiabatic stability analyses.

\begin{tabular}{llllll}
\hline Seq. & $M_{*} / M_{\odot}$ & $\Delta M_{\mathrm{He}} / M_{*}$ & $\log T_{\text {eff }}$ & models & $Z$ \\
\hline I5 & 0.5 & $1.5(-2)$ & $4.51-4.26$ & 12 & 0 \\
I6 & 0.6 & $1.5(-2)$ & $4.49-4.27$ & 30 & 0 \\
I7 & 0.7 & $1.5(-2)$ & $4.51-4.26$ & 12 & 0 \\
II & 0.6 & $1.5(-2)$ & $4.45-4.22$ & 16 & 0.02 \\
III & 0.6 & $6.6(-4)$ & $4.45-4.24$ & 21 & $Z_{\mathrm{CO}}$ \\
IV & 0.6 & $2.0(-6)$ & $4.46-4.27$ & 18 & $Z_{\mathrm{CO}}$ \\
\hline
\end{tabular}

essentially non-degenerate. In models b and c, on the other hand, the elemental depletion/enhancement region is already partially degenerate so that the temperature structure, which is influenced by the composition dependent opacity changes, plays only a minor role in the functional form of $\mathrm{d} \ln \rho / \mathrm{d} \ln r$ which enters the Brunt-Väisälä frequency computations.

The AGB evolutionary phase left its traces in the structure of the $\mathrm{C}$ and $\mathrm{O}$ profiles (Fig. 2) deep in the star and in particular in the gradients seen at $-\log q=0.3$ and about 1.1. The corresponding bumps in the BruntVäisälä frequencies are clearly discernible in all the three models shown in Fig. 3. The additional narrow BruntVäisälä frequency peak pointed at by vertical arrows in models a, b, and c are numerical artifacts: The switching between different numerical treatments of the EoS in the model-star computations introduced a small glitch in $\Gamma_{1}$ which eventually translated into a narrow spike in the Brunt-Väisälä frequency. The relevance of this spike is further addressed in Sect. 4. The teeth-like structure in the Brunt-Väisälä frequency between $5.5<-\log q<6.0$ of model a in Fig. 3 is the result of the switching between different compositions of tabular opacity data in the region of rapid spatial $\mathrm{C}$ and $\mathrm{O}$ change. Since this feature was restricted to the hottest models for which the corresponding temperature range was nondegenerate, because the feature occurred close enough to the surface to not interfere with mode trapping, and since it lay deep enough to not influence the driving/damping of the white dwarfs' pulsation we tolerated what is a cosmetic flaw in this case.

Table 1 lists the model sequences that are to be discussed in this paper. Except for the sequence I, only $0.6 M_{\odot}$ models were considered. To study the effect of mass on the instability domain of model stars devoid of heavy-elements, series I - with a helium envelope of $0.015 M_{*}-$ was computed for $0.5,0.6$, and $0.7 M_{\odot}$ each. The effect of heavy-elements was studied in sequence II, adopting $Z=0.02$. Both sequences, I and II, were computed without abundance diffusion which was included, however, in sequences III and IV. The latter two model series had also considerably shallower helium envelope masses with $6.6 \times 10^{-4} M_{*}$ and $2.0 \times 10^{-6} M_{*}$. With $Z_{\mathrm{CO}}$ we denote the metalicity calculated from the variable composition resulting from diffusion as $\min \left(0.02, X_{\mathrm{C}}+X_{\mathrm{O}}\right)$, with $X_{\mathrm{C}}$ and $X_{\mathrm{O}}$ standing for the mass fractions of $\mathrm{C}$ and $\mathrm{O}$. The functional form of the helium-gradient region in the diffusion-less sequences I and II is comparable to the initial one in Fig. 2 and it remains invariant under time evolution. Columns 4 and 5 list the range of effective temperatures covered by the pulsation-model sequences and the number of star models therein; defining hence roughly the achieved temperature resolutions in the instability domains.

\subsection{Initial models for diffusive sequences}

For this study, the pre-WD evolutionary phases were not modeled in detail. Rather, we obtained our initial models by artificially brightening an initial WD configuration (see Benvenuto \& Althaus 1998) up to high stellar luminosities. Specifically, we added an artificial, constant specific energy generation to the entire model. The energy was increased progressively until the stellar model reached the desired luminosity, which was much higher than that of the initial models considered here. Thereafter, the energy source was turned off smoothly. This procedure leads to an initial sequence of unphysical models which eventually settles onto the correct cooling curve long before the DBV instability strip is reached.

The core chemical composition of our models is that predicted by evolutionary calculations of WD progenitors (Salaris et al. 1997) and the mass of the helium envelope was varied in the range of $2 \times 10^{-6} \lesssim M_{\mathrm{He}} / M_{*} \lesssim$ $1.5 \times 10^{-2}$. Figure 2 shows, with heavy lines, the chemical profile of the starting model of model sequence III. The functional form the profiles is the same also for sequence IV. The envelope is characterized by mass abundances of helium, carbon and oxygen of $0.42,0.37$, and 0.21 , respectively. The outer layers' chemical composition we assumed for the starting models of sequences III and IV was taken from Herwig et al. (1999) who have followed the evolution of a post-AGB remnant through the very late thermal pulse it experienced on the early cooling branch (for the born-again scenario see Iben et al. 1983). On the basis of a detailed treatment in which nuclear burning and mixing are treated simultaneously, Herwig et al. (1999) obtained hydrogen-deficient models with C-O surface abundance in good agreement with observed abundances in PG 1159 stars. In view of recent studies (Unglaub \& Bues 2000), which indicate that mass loss in hot WDs prevents or retards gravitational settling of heavy elements; in our evolutionary models, diffusion is operative after the effective temperature dropped below $60000 \mathrm{~K}$.

\subsection{Pulsation treatment}

The linear nonadiabatic oscillation computations were performed with the same Riccati shooting-method as referred to in Gautschy et al. (1996). The complex eigenfrequencies, $\sigma$, are expressed in units of $\sqrt{3 G M_{*} / R_{*}^{3}}$, the "free-fall frequency" of the star. The fact that the Riccati approach is a direct integration of the nonadiabatic equations removes the numerical problem of linear 


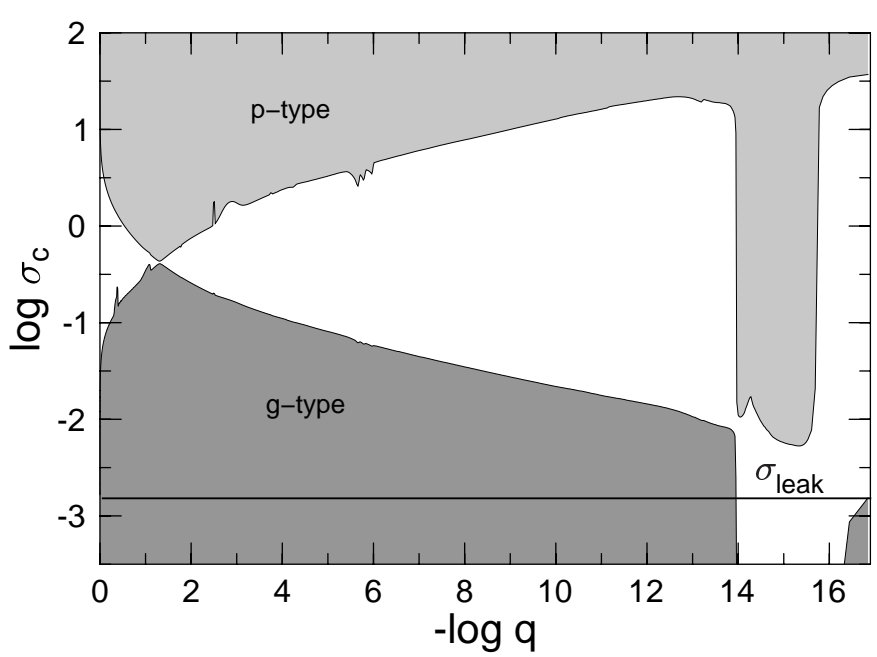

Fig. 4. The propagation diagram for plane waves for model a which is also shown in Fig. 2. The dark grey region shows where waves have $g$-type character, $p$-type waves propagate in the paler grey domain. The horizonal line indicating $\sigma_{\text {leak }}$ indicates the frequency below which $g$ modes are no longer fully reflected at the stellar surface.

dependencies between the nonadiabatic equations when they enter near-adiabaticity in the stellar interior. In other words, linear dependent equations can be directly integrated with the Riccati method whereas they cause singular matrices in finite difference schemes. The Riccati approach has already proven its suitability for white-dwarf pulsation studies before (Gautschy et al. 1996; Gautschy 1997). The full set of nonadiabatic equations was solved from the surface to the very center of the model stars. The interior boundary conditions were dictated by the central regularity of the solutions. At the surface we imposed a reflecting boundary and we assumed the validity of the linearized Stefan-Boltzmann law. Figure 4 shows the spatial propagation zones of plane waves as grey-shaded regions. Dark grey indicates $g$-type character of a wave and the paler grey $p$-type character. The critical frequencies were computed from the adiabatic Cowling equations with our choice of variables. The normalized critical frequency, $\sigma_{\text {leak }}$, below which $g$ modes leak into the atmosphere stayed in the range of $1.5-3.0 \times 10^{-3}$ for dipole modes of the $0.6 M_{\odot}$ model sequences. Depending on the position along the cooling track, the lowest $\sigma_{\text {leak }}$ translate into periods between 3300 and about $7000 \mathrm{~s}$. These numbers agree, within a factor of two, with those suggested in the analysis of Hansen et al. (1985). The longest periods considered in our computations remained always below the critical period at which leakage sets in. But even eigenmodes with $\sigma<\sigma_{\text {leak }}$ showed only slightly enhanced damping when running-wave boundary conditions were adopted at the surface. The reason can be attributed to the surface convection zone constituting an effective reflecting layer.

No pulsation-convection coupling was included in the computations. Therefore, all red boundaries of instability domains appearing in this paper must not be trusted.

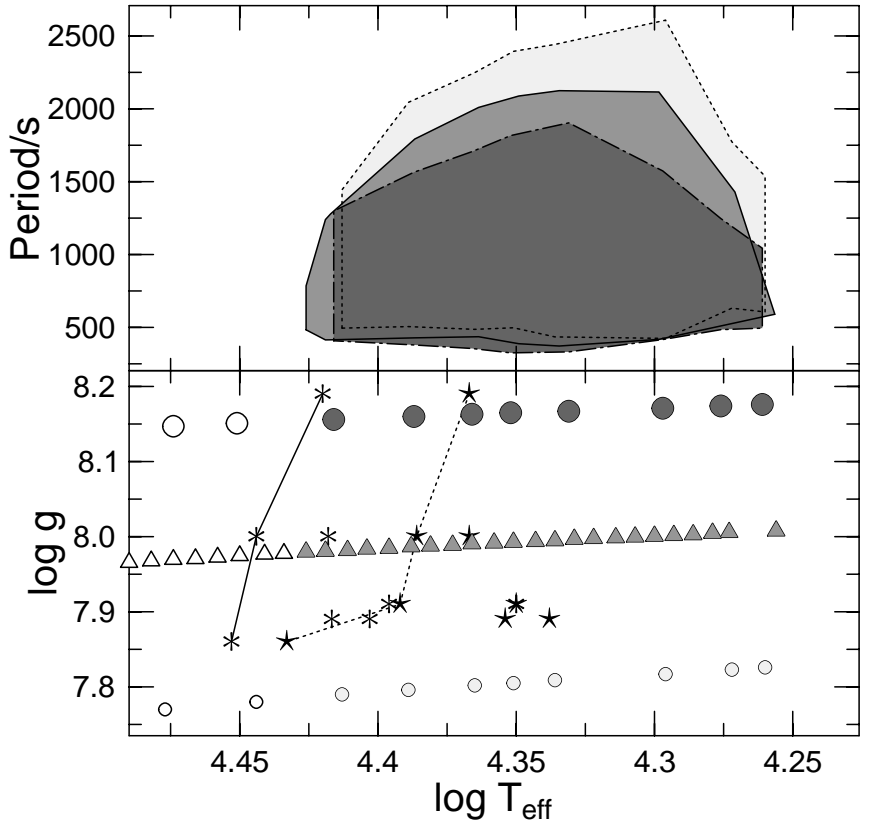

Fig. 5. Top panel: instability domain of dipole modes on the $\log T_{\text {eff }}$-period plane for the diffusion-free model sequences devoid of heavy elements for masses of $0.5 M_{\odot}$ (dark grey), $0.6 M_{\odot}$ (medium grey), and $0.7 M_{\odot}$ (light grey). Lower panel: loci of the model sequences on the $\log T_{\text {eff }}-\log g$ plane and their comparison with observed DBVs. Open symbols indicate pulsationally stable models, filled symbols stand for pulsational instability. Asterisks denote the calibration of the variable stars with hydrogen-free model atmospheres; the calibrations with some H traces left (cf. Beauchamp et al. 1999) are plotted as stars. The corresponding observational blue edges are indicated by full and dashed lines, respectively.

Possible forms of the influence of convection and its potential role played in DBVs are specifically addressed in Sect. 4.

The oscillation computations of most model sequences had to be terminated at around $\log T_{\text {eff }}=4.25$ despite the evolutionary sequences extending to lower temperatures. The use of tabular EoS data to compute the high-density stellar interiors caused increasingly spiky spatial density gradients which in turn introduced intolerable noise in the spatial run of the Brunt-Väisälä frequency.

\section{The DB instability}

In this section, we present the results from stability analyses of the model sequences listed in Table 1. Nonadiabatic oscillation spectra were computed for $\ell=1$ and $\ell=2$ $g$ modes in the period range between 100 and about $2800 \mathrm{~s}$. If not stated otherwise, the data are presented for the observationally preferred dipole modes only. We begin with the non-diffusive models, comparing $g$-mode instability of models with $Z=0$ with $Z=2 \times 10^{-2}$ ones. Most of the section is devoted, however, to the instability domains and trapping properties of those DB models computed with diffusion. 


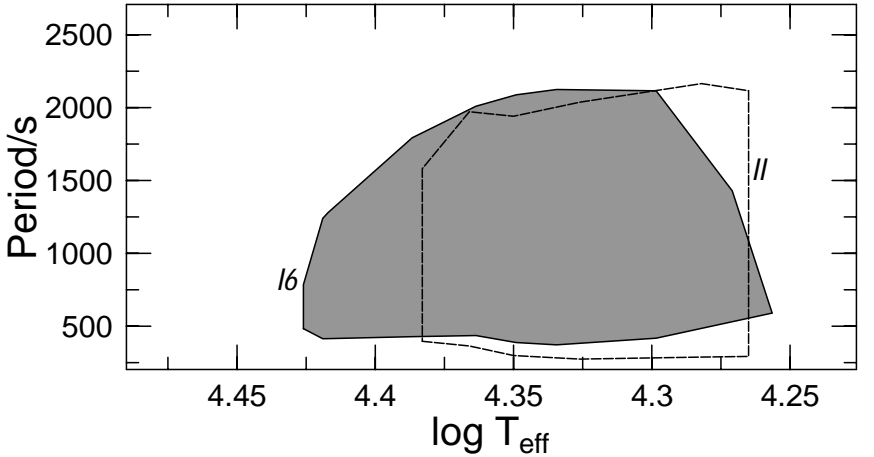

Fig. 6. Dipole-mode instability domains on the $\log T_{\text {eff- }}$-period plane of diffusion-free $0.6 M_{\odot}$ model sequences devoid of heavy elements (dark grey area; I6) and with $Z=2 \times 10^{-2}$ (light grey area; II).

The top panel of Fig. 5 illustrates the extents of the dipole $g$-mode instability domains of sequences I5 (dark grey), I6 (medium grey), and I7 (light grey). Within the given model density, the blue edge of the instability domains shows no mass dependence and lies at about $\log T_{\text {eff }}=4.42$. Only for sequence I6 we encountered the red edge in the last model of the sequence at about $\log T_{\text {eff }}=4.25$. The other two model sequences I5 and I7, show a strong shrinking of the excitation rates and a rapid decrease of the number of overstable $g$ modes towards $\log T_{\text {eff }}=4.25$; the cool instability boundary was not yet reached, however. Of the excited modes, the minimum period dropped from $424 \mathrm{~s}$ in I5, to $372 \mathrm{~s}$ in I6, to finally $335 \mathrm{~s}$ in I7. The maximum period, on the other hand dropped in the same order from $2608 \mathrm{~s}$, to $2124 \mathrm{~s}$, and $1875 \mathrm{~s}$. The minimum as well as maximum periods were encountered between $4.30<\log T_{\text {eff }}<4.33$, this means that at most 45 (41) dipole modes were excited in sequence I5 (I6 and I7).

The bottom panel of Fig. 5 compares the loci of the model sequences I5, I6, and I7 on the $\log g-\log T_{\text {eff }}$ plane with observed DBV stars as adapted from Beauchamp et al. (1999). Open symbols stand for pulsationally stable models and filled ones for overstable models. We code I5 with small circles, I6 with triangles, and I7 with large circles. Observations are plotted as stars and asterisks. The star symbols show the results from fitting model atmospheres to observations by neglecting the presence of hydrogen. The systematically cooler calibrations included traces of hydrogen that did not yet contradict the observed spectra. The corresponding "observational blue edges" are plotted as solid and dashed lines, respectively. Since the uncertainties in $\log g$ are a few hundredth of a dex only, the divergence of the full and the dashed lines measure the uncertainty of the blue-edge position along the effectivetemperature axis.

\subsection{Heavy elements and the $D B$ instability strip}

The dipole instability domains of model sequences I6 and II are displayed in Fig. 6. Interestingly enough, compared with I6, the dipole blue edge of sequence II is about

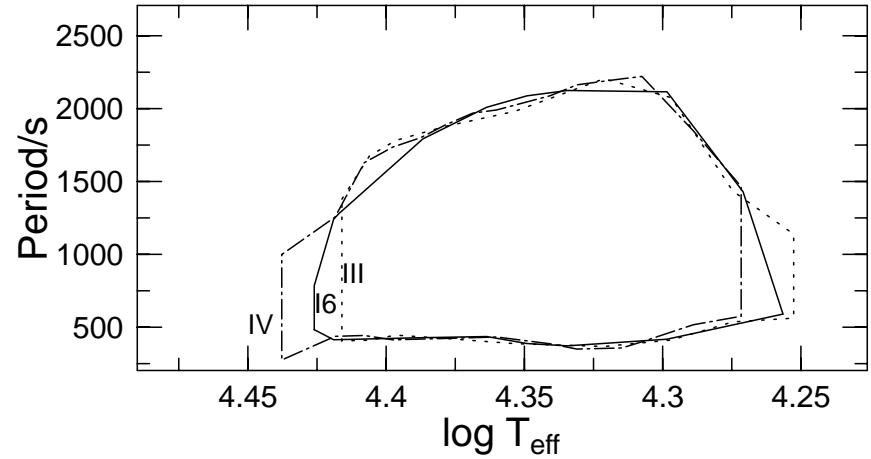

Fig. 7. Instability domains on the $\log T_{\text {eff }}$-period plane of model sequences I6, III, and IV.

$2500 \mathrm{~K}$ cooler. At the low- $T_{\text {eff }}$ boundary of sequence II, i.e. at about $\log T_{\text {eff }}=4.27$, the instability domain does not yet show signs of the proximity of its red edge. Compared with sequence I6, II has both longer and shorter periods excited at low effective temperatures. The periods of the overstable lowest-order modes are as short as 275 s. Hence, the instability domains of I6 and II are not only laterally in $T_{\text {eff }}$ - shifted, but the low-period border of sequence II lies significantly below the one of sequence I6. According to our computations, any remaining traces of heavy elements, at least up to solar, in DB envelopes do not enhance pulsational instability and shift the blue edge to higher effective temperatures. Just the contrary occurs, the blue edge shifts to lower effective temperature. The presence of heavy elements in the stellar matter reduces the thermal timescale of the envelope. Compared with $Z=0$ models, the $Z=0.02$ ones must get cooler (for more mass to lie above the driving layers) before a particular pulsation mode is destabilized.

\subsection{Helium-envelope thickness and the DB instability strip}

Figure 7 displays the boundaries of the dipole instability domains of sequences of $0.6 M_{\odot}$ with different heliumenvelope masses. Sequence I6 with its $0.015 M_{*}$ of superficial helium and no diffusion acting led to the instability domain shown by a full line. The instability borders of model sequence III - with $6.6 \times 10^{-4} M_{*}$ of helium on the surface - is plotted with a dotted line. It should be stressed that not the whole period range of sequence III between 500 and $1200 \mathrm{~s}$ is overstable at the red boundary at $\log T_{\text {eff }}=4.25$. Due to mode trapping, which is discussed in more detail below, only a few preferred modes are driven overstable close to the boundaries of the instability domain (for a conceptual elucidation see e.g. Fig. 8). The blue edge is encountered at $\log T_{\text {eff }}=4.416$, it is the coolest of the three model families being compared here. Eventually, series IV with the thinnest helium blanket $\left(2 \times 10^{-6} M_{*}\right)$ resulted in the instability domain which is traced out by the dash-dotted line.

Independent of diffusion and the thickness of the helium envelope, the loci of the shortest and the longest 


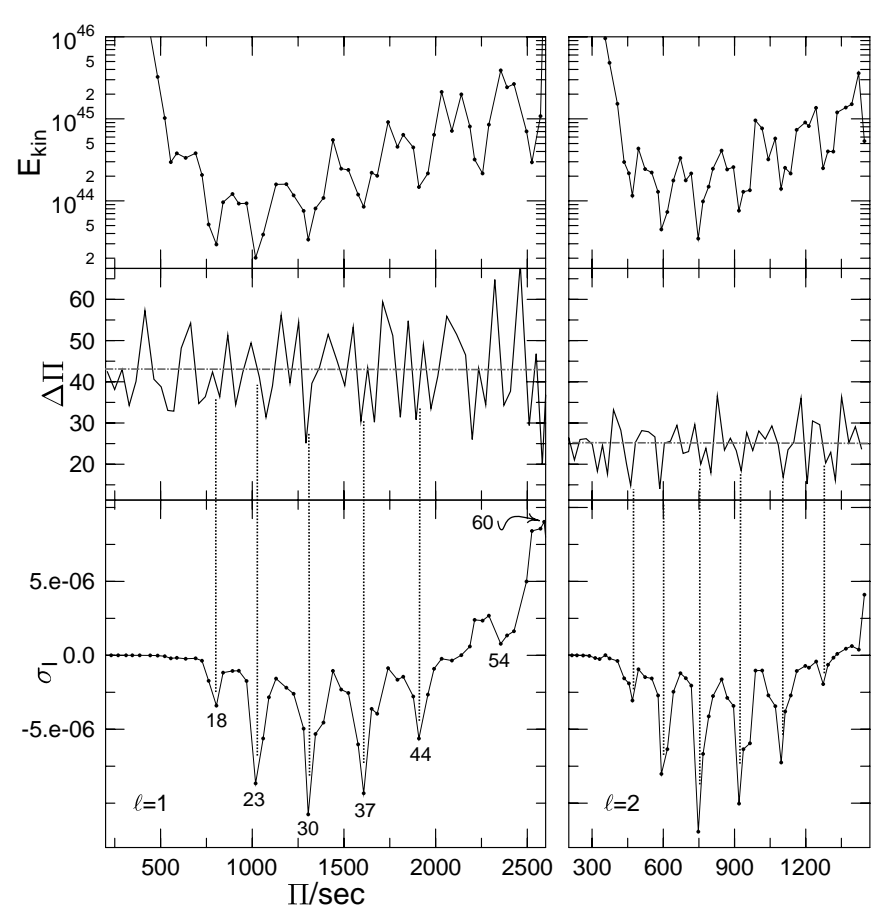

Fig. 8. Eigendata for dipole (left panels) and quadrupole modes (right panels) of a sequence IV model star at $\log T_{\text {eff }}=$ 4.341. Top panels: integrated kinetic energies of the oscillation modes as a function of period. Cyclical trapping of modes appears as local minima in the total kinetic energy $E_{\text {kin }}$. Middle panels: period separations as a function of period. Horizontal dotted lines delineate the asymptotic period separation of the respective $g$ modes. Lower panels: imaginary parts of the freefall normalized eigenvalues. Negative numbers denote pulsationally overstable modes, positive ones damped modes. Notice the well pronounced cyclically enhanced driving and damping, respectively. The numbers at the peaks of $\sigma_{\text {I }}$ label the radial orders, $k$, of the corresponding eigenmodes.

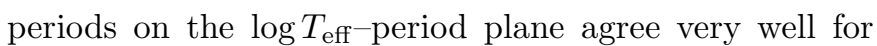
the three sequences I6, III, and IV. The blue edges, on the other hand differ. The skinniest He-envelope models, those of series IV, show the hottest blue edge at $\log T_{\text {eff }}=4.438$. The diffusion-less sequence I6 with the thickest He mass floating on the surface marks the intermediate position of the blue edge at $\log T_{\text {eff }}=4.416$. Finally, for the diffusive sequence III, the instability domain starts at $\log T_{\text {eff }}=4.416$. The temperature difference of the blue-edge locations between sequences III and IV amounts to about $1350 \mathrm{~K}$.

\subsection{Mode-trapping in diffusive models}

Mode trapping in compositionally stratified white dwarfs is asteroseismologically well taken advantage of (e.g. Bradley \& Winget 1994 and references therein) and thoroughly investigated in adiabatic pulsation theory (Brassard et al. 1992). We restrict our presentation therefore to aspects of nonadiabaticity and discuss selected models for the sake of clarity and not for their suitability to fit a particular object.
For the model star at $\log T_{\text {eff }}=4.341$ of model sequence IV, Fig. 8 shows the period separation, $\Delta \Pi$, between adjacent radial orders for $\ell=1$ in the mid-left panel and for $\ell=2$ on the mid-right. The period separation is defined by the "forward difference": $(\Delta \Pi)_{k} \equiv \Pi_{k+1}-\Pi_{k}$. The dotted horizontal lines in both panels indicate the period separation as computed from asymptotic theory: $43.6 \mathrm{~s}$ for $\ell=1$ and $25.2 \mathrm{~s}$ for $\ell=2$, respectively. The cycle length varies roughly symmetrically about the asymptotic separation. Some cyclical components seem to be contained in both middle panels; the magnitude of the period separation prevents, however, a more detailed quantification.

The "trapping amplitude", i.e. the magnitude of the variation of $\Delta \Pi$ as function of $\Pi$ is about $35 \mathrm{~s}$ for $\ell=1$ and $20 \mathrm{~s}$ for $\ell=2$. These numbers are somewhat bigger than what is needed for example for GD 358 (cf. Bradley \& Winget 1994); nevertheless, the qualitative character of the period separation $\Delta \Pi$ as a function of $\Pi$ compares well with other studies.

The lower panels of Fig. 8 contain the imaginary parts of the eigenfrequencies, expressed in units of the star's free-fall frequency. The black dots mark the actual positions of the discrete eigenvalues. Negative values indicate an oscillatory overstability, positive values mark damped modes. In the model shown, oscillatory variability prevails between 385 and 2089 s. In contrast to the period separations, the imaginary parts exhibit a pronounced and clearly defined cyclic variability in the sense that in the instability domain, enhanced instability and in the stability range enhanced damping is observed for selected radial overtones. The cycle length inherent in the imaginary parts is clearly longer than in the period separation; this is further visualized with the vertical lines connecting the instability spikes with the period separation diagram. Neither for $\ell=1$ nor for $\ell=2$ do we see any peculiar or systematic behavior of $\Delta \Pi$ at the locations of enhanced (in)stability. In particular, not all of $\sigma_{\mathrm{I}}$-enhanced modes do correspond to minima in $\Delta \Pi$. The trappingcycle length has, both for dipole and quadrupole modes, a stride $\Delta k=7$, except at low periods where it shrinks to $\Delta k=5$ in both cases.

The total kinetic energy of the oscillation modes is shown in the top panels of Fig. 8. Evidently, the enhanced excitation and damping rates of the bottom panels correlate with the local minima of the kinetic energies which go along with the envelope trapping of eigenmodes.

Figure 9 quantifies the cycle lengths in the $\sigma_{\text {I }}$ vs. $\Pi$ of sequences III and IV. Sequence III is referred to by circles and sequence IV by triangles. Dipole-mode results are plotted as open symbols, those for quadrupole modes are shown as filled symbols. The magnitude of the separations between the peaks of the imaginary parts of the eigenfrequencies show a clear dependence on the model sequence, i.e. on the envelope helium-layer thickness. The cycle length $\Pi_{\text {trap }}$ [imag] varies between about 80 and $165 \mathrm{~s}$ for sequence III's dipole modes and between 260 and $350 \mathrm{~s}$ in sequence IV. For the quadrupole modes the cycle lengths 


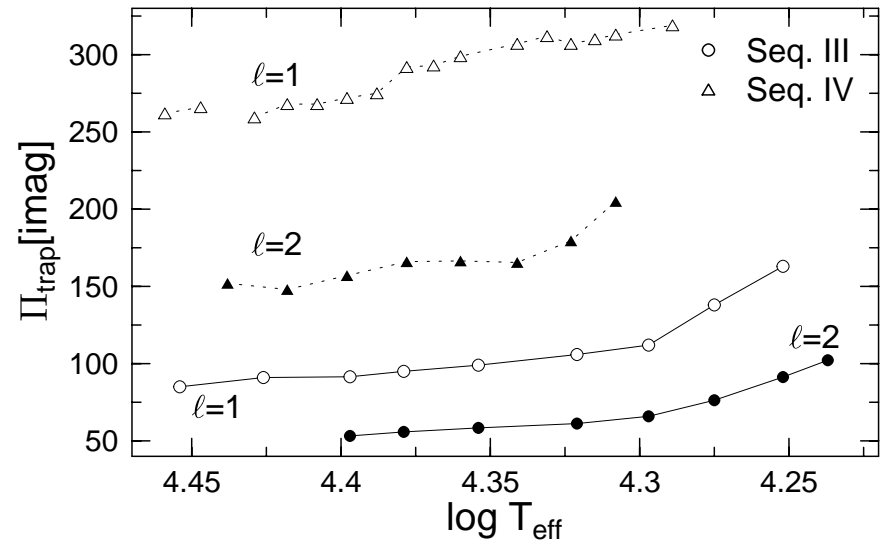

Fig. 9. Trapping-cycle length as measured in the imaginary parts of the eigenvalue data as a function of effective temperature of the model sequences III and IV.

are again systematically shorter: between 50 and $100 \mathrm{~s}$ for sequence III and between 150 and $200 \mathrm{~s}$ for sequence IV. The ratio $\Pi_{\text {trap }}[\operatorname{imag}](\ell=1) / \Pi_{\text {trap }}[\mathrm{imag}](\ell=2)$ varies between 1.5 and 1.83 for sequence IV, whereas it stays rather constant at around 1.75 for sequence III.

\section{Discussion}

To compare the instability domains from our computations with the corresponding distribution of DBVs in nature, we rely on the spectroscopic calibrations of Beauchamp et al. (1999). Our models are all hydrogen free, therefore the correspondingly higher $T_{\text {eff }}-$ scale deduced for hydrogen-free atmospheres in Beauchamp et al. are more relevant. Further evidence for a high- $T_{\text {eff }}$ temperature scale comes from the spectroscopic study of $\mathrm{C}$ and $\mathrm{H}$ abundances in DBs by Provencal et al. (2000). The period information was taken from Bradley (2000) plus the new data on PG $2246+121$ as published by Handler (2001).

Only sequence IV had a blue edge hot enough for the dipole instability domain to safely enclose most of the observed DBVs. In particular on the hot side of the instability domain (EC 20058-5234, PG 1654+160, and PG 2246+121), the DBVs appear to pulsate with rather short periods. Dipole instabilities alone, as computed by us, cannot explain such short periods. Therefore, Fig. 10 shows with a grey inlet also the extent of the quadrupole overstability region for sequence IV.

Using the high-temperature scale to calibrate the observed DBVs, we see that sequence IV falls only slightly short in enclosing their instability region on the $\log T_{\text {eff- }}-$ period plane: EC 20058-5234 and PG 1654+160 are slightly hotter than sequence IV's instability domains. With a simple short-ranged blueward extrapolation, the observed period ranges can, however, be incorporated into these instability domains. The results of sequence III indicated that the overstable shorter-period quadrupole modes have their blue edge at higher $T_{\text {eff }}$ s than the dipole modes. Therefore, despite the current lack of observational

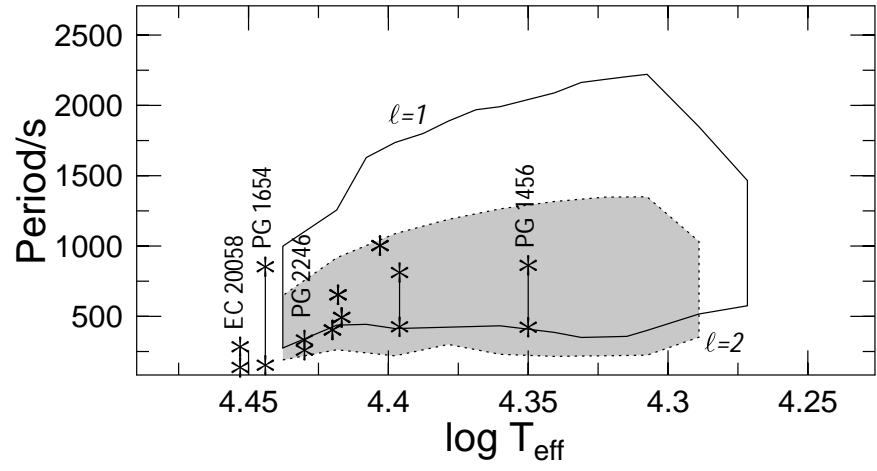

Fig. 10. Dipole and quadrupole instability domain for model sequence IV. Observed DBVs are plotted as asterisks. Period ranges for selected variables are indicated by vertical lines. Stars of particular interest are additionally labeled.

evidence for $\ell=2$ modes, according to our computations the shortest periods of the hottest DBVs appear to be good candidates for quadrupole modes.

Measured again with the high-temperature scale of Beauchamp et al. (1999), most of the observed DBVs have effective temperatures exceeding $\log T_{\text {eff }}=4.4$. One exception is $\mathrm{PG} 1456+160$ at $\log T_{\text {eff }} \approx 4.35$ which is classified as a DBA variable with remaining hydrogen traces in its envelope. Therefore, it is not a reliable pillar to discuss the extent of the instability strip. Even if we included PG 1456+160 blindly, one big discrepancy between our computations and the observations remains evident: the computed instability domain extends to much too low temperatures, i.e. the red boundary is much too cool. Our modeling seemingly misses a significant dissipation agent in the stellar envelopes. As a star's effective temperature drops, the driving region moves deeper in mass. Hence, the crucial thermo-mechanical interaction time-scale grows. Therefore, we seem to have missed a dissipation agent acting on long time-scales.

As criticized a long time ago by Brickhill (1990), also we continue to stagnate with the observation that the instability region is not only too extended along the $T_{\text {eff }}$ axis but also in the second direction on the $T_{\text {eff }}$-period plane. Except close to the blue edge, much longer periods are computed to be excited than what is observed and the discrepancy grows towards lower effective temperatures. In the worst case, about a factor of two too long periods are found overstable in the computations. The discrepancy cannot be attributed to a missed inclusion of wave leakage into the atmosphere. In all cases, the longest overstable periods lie well below the critical periods above which waves are no longer well reflected at the stellar surface. The short-period boundary of the instability domain agrees, on the other hand, decently well with observations. Hence, as encountered along the $T_{\text {eff }}$ axis, the missing dissipation seems to act on long time-scales of $1000 \mathrm{~s}$ and above. For our CGM-convection based white-dwarf models, convective damping is more urgently needed than a source of convective driving. 
The existing literature (Bradley \& Winget 1994; Beauchamp et al. 1999) teaches us that the DBV blue edge gets hotter with increasing efficiency of the convective mixing-length dialect adopted for the stellar modeling. Choosing $\log g=8.0$ which is the most relevant surface gravity for us, Beauchamp et al. (1999) computed the blue edges at $\log T_{\text {eff }}=4.33,4.43$, and 4.49 when using ML1, ML2, and ML3, respectively. Hence, the dipole blue edges of our CGM-based white-dwarf models lie close to the one of ML2 models in the Beauchamp et al. study. An exception is sequence II, for which pulsational instability sets in at lower effective temperatures, translating into a less efficient mixing-length convection picture. Comparing our CGM results with the Bradley \& Winget (1994) ML-results favor, on the other hand, the high efficiency of ML3. This is caused by the systematically cooler blue edges found by Bradley \& Winget as compared with Beauchamp et al. (1999).

Any discussion of the location of the instability domain of white dwarfs is unsatisfactory and must remain suspect as long as the role played by convection is not appropriately incorporated. In contrast to the DAV study of Gautschy et al. (1996) this project is a retrogression since no simulation data were available on the divergence of the perturbed convective flux. Furthermore, we were unable to implement a credible and robust scheme for pulsation convection interaction. Therefore, the instability-domain discussion, in particular aspects concerning the red edge, must be taken cum grano salis. Nevertheless, the models computed with convection based on the CGM formalism show the onset of pulsational instability at effective temperatures comparable to spectroscopic calibrations of DBVs in nature.

The non-uniformity of the period separations which we encountered in the computations compares well with the results in the extensive literature on mode trapping in compositionally stratified white dwarfs. The novel aspect contained in this study is the striking difference in trapping behavior between period separation and damping rates as a function of period (cf. Fig. 8).

The local minima of $\sigma_{\mathrm{I}}$ are associated with clear minima in the kinetic energies of the corresponding eigenmodes. As discussed e.g. in Brassard et al. (1992), such modes are trapped in the superficial layers of a compositionally stratified star and they have reduced amplitudes in the deep stellar interior. The top panel of Fig. 11 shows the kinetic-energy density of two overstable dipole modes; the $k=30$ mode is clearly confined to the helium-rich superficial layers of the star. The mode with radial order $k=26$, on the other hand, shows an enhancement of kinetic energy density in the $\mathrm{C}$ - and O-rich interior over the contributions from the superficial regions. For all modes, we found the trapping (as seen in the imaginary parts of the eigenfrequencies) to occur at the upper edge of the diffusive depletion region of helium, i.e. the diffusive tail into the stellar interior does not seem to be important for the trapping spectrum of the star.

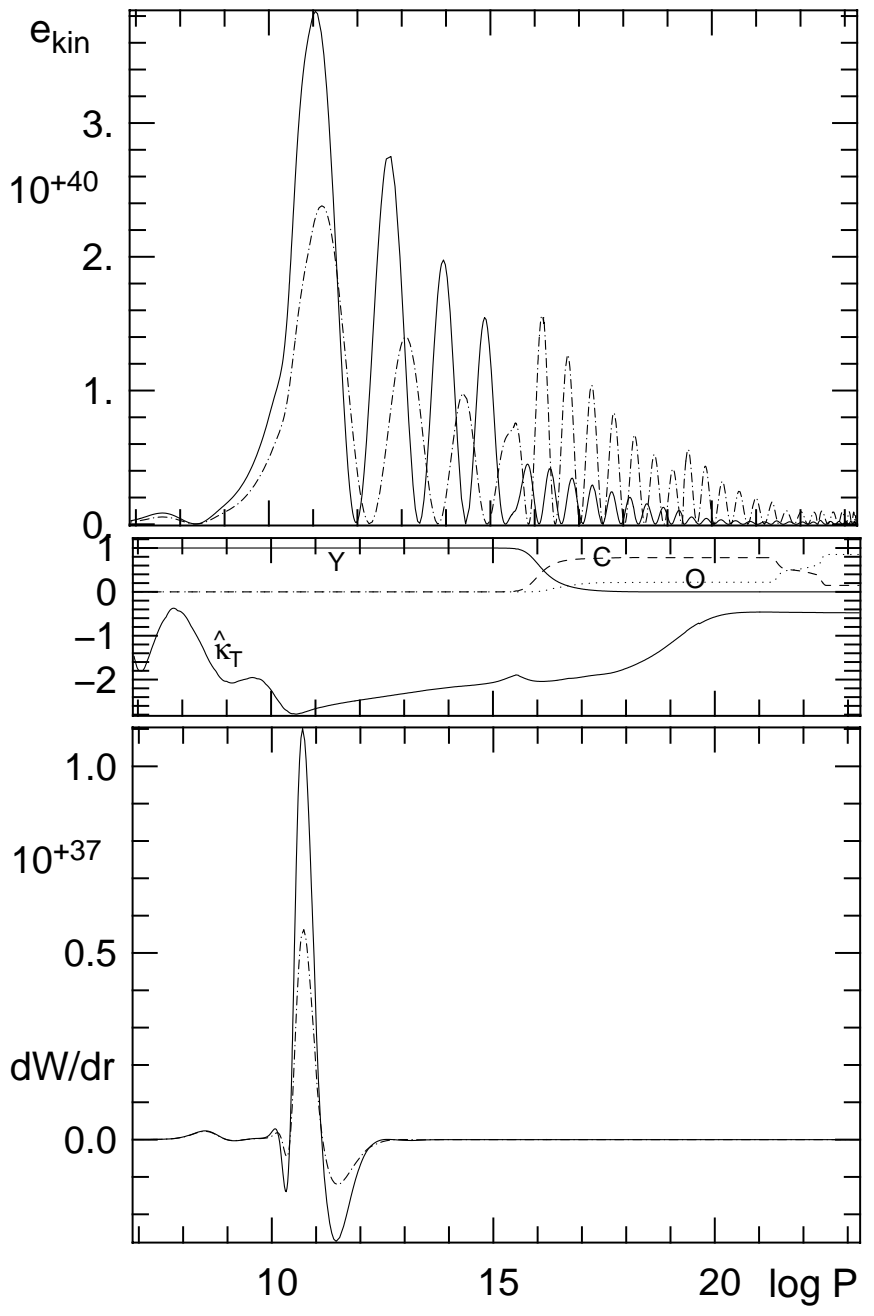

Fig. 11. Comparison of eigenmode properties for dipole modes of radial order $k=26$ (dash-dotted line) and $k=30$ (full line) of model at $\log T_{\text {eff }}=4.341$ of sequence IV. The top panel shows the kinetic energy densities. The middle panel plots structural quantities: the abundances of helium, carbon, and oxygen, together with $\hat{\kappa_{\mathrm{T}}}=\kappa_{\mathrm{T}} / 5-1$. The bottom panel displays the differential work done by the eigenmodes.

Enhanced instabilities were found to go with trapping of the corresponding modes in the helium-rich surface layers; quenched instability was found for modes with reduced kinetic energy density in the pure helium surface and substantial kinetic energy in the CO interior. This behavior is mirrored to the damped region: modes with stronger confinement to the $\mathrm{CO}$ interior (e.g. $k=54$ in Fig. 8) show reduced, the superficially trapped ones (e.g. $k=60$ in Fig. 8) experience enhanced damping.

The work integrals of the various modes hint indeed at the phase relations between say pressure and density perturbation to be modulated cyclically by the trapping process. Correspondingly, favorable phasing induces enhanced driving and detuning in the very same layers causes enhanced damping. The bottom panel in Fig. 11 supports this picture. Compared with mode $k=26$, the superficially trapped one with $k=30$ shows enhanced radiative 
damping below the opacity peak due to ground-state transitions in $\mathrm{He}^{+}$(at about $\log T \approx 5.2$ which corresponds to $\log P \approx 11.5$ in the model shown) and enhanced driving in the opacity peak region. All the important contributions to the work integral come from the layers above the first node of the displacement eigencomponent. The deeper lying particularities of the solution are hence of no direct influence.

It is worthwhile to point out once more that the periodseparation diagrams display a considerably more complicated trapping structure than the $\sigma_{\mathrm{I}}-$ period diagrams. As discussed before, the $\sigma_{\mathrm{I}} \mathrm{s}$ react essentially on trapping induced by the top of the helium diffusion layer only. The periods on the other hand react also on partial trapping occurring in the deep stellar interior. Two additional trapping layers are formed by the molecular weight gradients caused by the $\mathrm{C}$ and $\mathrm{O}$ abundance changes located at $-\log q=0.3$ and 1.1 in Fig. 2. The kinetic energy of the modes and therefore also the weight functions react on these deeply buried trapping layers. The many eigenfunction nodes above these trapping layers and the near adiabaticity of the problem at the trapping depth do not alter, on the other hand, inter-component phase relations sufficiently strong to cause a significant modulation of the

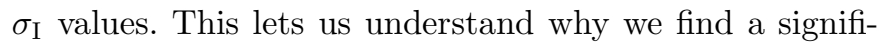
cantly reduced trapping amplitude in $\sigma_{\mathrm{I}}$ for models with thick helium layers (such as sequences I and II).

Finally, we must caution from over-interpreting the details in the period-separation diagram of Fig. 8. The $\Gamma_{1}$ glitch mentioned in Sect. 2.1 caused an additional - unreal - partial trapping of pulsation modes. The trapping length, $\Delta k$, induced by the glitch is about 2.6 (cf. Brassard et al. 1992; their Eq. (43)). The two deep-lying trapping features addressed in the last paragraph (and clearly visible in the Brunt-Väisälä frequency in Fig. 3) cause shorter trapping lengths of 1.6 and 1.3 cycles, respectively. These $\Delta k$ numbers explain the wiggly $\Delta \Pi$ behavior on a scale as short as the period separation of two consecutive eigenmodes of like degree.

\section{Conclusions}

The instability domains of the diffusion-free models with nonvanishing heavy-element abundances in their envelopes were not strongly shifted relative to those of models with pure helium envelopes. If we push our results to their limits, there seems to be an indication that contrary to our initial expectations - stars with heavyelement enriched envelopes seem to develop oscillatory instabilities at lower effective temperatures than stars with pure helium ones.

The diffusion-induced abundance gradients in the model stars appear to be shallower than many analytical prescriptions used hitherto. The main effect of profiles resulting from consistent diffusion modeling is a weaker trapping effect on the periodicities. On the other hand, the trapping effect is well conserved and expressed in the imaginary parts of the eigenfrequencies as long as the pure
He envelope stays shallow enough. Trapping at the He gradient is effective only if the pure He envelope is shallow enough for the diffusive He depletion to set in where the stellar matter is still essentially non-degenerate. In the nondegenerate region, also the spatial run of the radiative opacity influences the trapping details. In model sequence IV, where the effect was well expressed, the mass of the pure He envelope was $1.9 \times 10^{-7} M_{\odot}$ at the blue edge of the instability domain. In sequence III, with a weaker imaginary-part trapping, the pure He envelope had a mass of $1.6 \times 10^{-6} M_{\odot}$ at the hot border of the instability domain.

Compared with observations, our computations based on CGM-convection star models - show the instability domains of dipole and quadrupole oscillations to have a much to large extension to the red. The blue-edge position is surprisingly good, however. Furthermore, as in the previous studies of this subject, much too long periods are excited in the model stars. Both findings hint at a missing dissipation agent in the computations. As we were unable to include a credible pulsation-convection interaction in the computations, we attribute the obvious shortcomings - in a long stellar physical tradition - to this deficiency. If the superficial convection zone of DB models as computed in this study has structural discrepancies like those discussed in Gautschy et al. (1996) for DAVs, then it is conceivable that also additional convective driving is eventually needed to arrive at an agreement with observational calibrations.

Lately, Brickhill's (1983, 1990) pulsation-convection picture was revitalized by Wu \& Goldreich (1999) to interpret the abundant data of the ZZ Ceti class. An application of the same formalism to DBVs was always taken for granted; we were, unfortunately, yet unable to incorporate it in our numerical Riccati scheme. Without a reliable pulsation - convection interaction scheme the location of the red edges for DBVs and DAVs cannot be modeled. Hence, concerning boundaries of instability domains, our computations constrain the location of the blue edges.

At the very least, the trapping results of our computations should remain valid even after a proper incorporation of pulsation-convection coupling: it is plausible that even after accounting for the action of a still elusive dissipation mechanism, only a fraction of the modes in the excitation peaks in $\sigma_{\mathrm{I}}$ survive eventually and therewith drastically reduce the number of excited modes and hence diminish the discrepancy between the computed number of excited and the actually observed modes. Hence, according to e.g. Fig. 8, we expect the formation of at most a few instability islands with only a few overstable consecutive modes separated from each other by stability gaps of the order of a few hundred seconds in the case of dipole modes.

The quadrupole modes we computed for this study showed instabilities comparable to those of dipole modes. The excited quadrupole modes have typically shorter periods than the dipole ones; the two domains overlap in the range of about $500<P<1000 \mathrm{~s}$. According to our computations, the period range between about 250 and $500 \mathrm{~s}$ 
belongs almost exclusively to quadrupole modes. The lack of observational evidence for any quadrupole modes in DBV data requires careful attention in the future.

Acknowledgements. A.G. is pleased to acknowledge financial support by the Austrian Fonds zur Förderung der Wissenschaften through grant S7305-AST. The convergence of S. Fendant and H. Saio in Basel a few years ago led to the conception of this project, A.G. is sincerely indebted to both of them. Additionally, we benefitted considerably from H. Saio's Coxian insights into stellar physics. This research has made use of NASA's Astrophysical Data System Abstract Service. Finally, the presentation of the paper profitted from an anonymous referee's constructive criticism.

\section{References}

Althaus, L. G., \& Benvenuto, O. G. 1997, ApJ, 477, 313

Althaus, L. G., \& Benvenuto, O. G. 2000, MNRAS, 317, 952

Althaus, L. G., Serenelli, A. M., \& Benvenuto, O. G. 2001, MNRAS, 323, 471

Beauchamp, A., Wesemael, F., Bergeron, P., et al. 1999, ApJ, 516,887

Benvenuto, O. G., \& Althaus, L. G. 1998, MNRAS, 293, 177

Bradley, P. A. 1996, ApJ, 468, 350

Bradley, P. A. 2000, Baltic Astron., 9, 485

Bradley, P. A., \& Winget, D. E. 1994, ApJ, 430, 850

Bradley, P. A., Winget, D. E., \& Wood, M. A. 1993, ApJ, 406, 661

Brassard, P., Fontaine, G., Wesemael, F., \& Hansen, C. J. 1992, ApJS, 80, 369

Brickhill, A. J. 1983, MNRAS, 204, 537

Brickhill, A. J. 1990, MNRAS, 246, 510

Burgers, J. M. 1969, Flow Equations for Composite Gases (New York: Academic Press)

Canuto, V. M., Goldman, I., \& Mazzitelli, I. 1996, ApJ, 473, 550
Dehner, B. T., \& Kawaler, S. D. 1995, ApJ, 445, L141

Gautschy, A. 1997, A\&A, 320, 811

Gautschy, A., \& Saio, H. 1996, ARA\&A, 34, 551

Gautschy, A., Ludwig, H.-G., \& Freytag, B. 1996, A\&A, 311, 493

Handler, G. 2001, MNRAS, 323, L43

Hansen, C. J., Winget, D. E., \& Kawaler, S. D. 1985, ApJ, 297, 544

Herwig, F., Blöcker, T., Langer, N., \& Driebe, T. 1999, A\&A, 349, L5

Iben, I. Jr., \& MacDonald, J. 1985, ApJ, 296, 540

Iben, I. Jr., Kaler, J. B., Truran, J. W., \& Renzini, A. 1983, ApJ, 264, 605

Iglesias, C. A., \& Rogers, F. J. 1996, ApJ, 464, 943

Kawaler, S. D. 1998, Baltic Astron., 7, 11

Kawaler, S. D., Sekii, T., \& Gough, D. 1999, ApJ, 516, 349

Kippenhahn, R., Weigert, A., \& Hofmeister, E. 1967, in Methods in computational physics, vol. 7, ed. B. Alder, S. Fernbach, \& M. Rottemberg (Academic Press, New York), 129

MacDonald, J., Hernanz, M., \& José, J. 1998, MNRAS, 296, 523

Magni, G., \& Mazzitelli, I. 1979, A\&A, 72, 134

Nather, R. E. 1995, Baltic Astron., 4, 117

Paquette, C., Pelletier, C., Fontaine, G., \& Michaud, G. 1986a, ApJS, 61, 177

Paquette, C., Pelletier, C., Fontaine, G., \& Michaud, G. 1986b, ApJS, 61, 197

Provencal, J. L., Shipman, H. L., Thejll, P., \& Vennes, S. 2000, ApJ, 542, 1041

Salaris, M., Domínguez, I., García-Berro, E., et al. 1997, ApJ, 486,413

Seaton, M. J., Yan, Y., Mihalas, D., \& Pradhan, A. K. 1994, MNRAS, 266, 805

Tassoul, M., Fontaine, G., \& Winget, D. 1990, ApJS, 72, 335

Unglaub, K., \& Bues, I. 2000, A\&A, 359, 1042

Wu, Y., \& Goldreich, P. 1999, ApJ, 511, 904 